\section{Invasive Neophyten in den Schweizer Mooren}

\author{
Meinrad Küchler
}

Eingegangen: 30. September 2009/

Online publiziert: 4. November 2009

Redaktion: Sabine Güsewell

Invasive Neophyten sind weltweit eine der grössten Bedrohungen für die Biodiversität. Besonders kritisch ist deren Ausbreitung in Habitatstypen, welche seltene und bedrohte Arten beherbergen, denn die lokale Verdrängung dieser Arten kann zu deren regionalem Aussterben führen. In der Schweiz gehören Moore zu den Habitatstypen mit einem besonders hohen Anteil seltener und bedrohter Arten. Entsprechend muss das Vorkommen invasiver Neophyten in Mooren besonders genau überwacht werden, um rechtzeitig regulierende Massnahmen zu ergreifen und eine massive Ausbreitung zu verhindern. Dabei ist eine Differenzierung zwischen Vegetationstypen sinnvoll, denn seltene, gefährdete Arten kommen primär in den nährstoffärmsten Moortypen vor.

Die vorliegende Untersuchung beschreibt die gegewärtige Verbreitung von invasiven Neophyten in Mooren der Schweiz. Sie soll eine Beurteilung ermöglichen, ob die Moore durch invasive Neophyten bedroht sind. Es stellen sich folgende Fragen:

- Welche invasive Neophyten kommen gegenwärtig in Mooren häufig vor?

- Welche Regionen der Schweiz sind betroffen?

- Bei welchen Standortsverhältnissen kommen invasive Neophyten vor?

- Welche Vegetationstypen der Moore sind betroffen?

Als Datengrundlage standen die Aufnahmen aus der Wirkungskontrolle Moorschutz (http://www.wsl.ch/forschung/ forschungsunits/oekologie/biotop/) zur Verfügung. Die

\section{Küchler $(\bowtie)$}

Eidgenössische Forschungsanstalt für Wald,

Schnee und Landschaft WSL, Zürcherstrasse 111,

8903 Birmensdorf, Switzerland

e-mail: meinrad.kuechler@wsl.ch
Daten stammen aus 112 Mooren und wurden in den Jahren 1996-2001 erhoben. Auch das engere Umfeld der Moorbiotope wurde mit Aufnahmen belegt. Wir bezeichnen die Moore mit ihrem engeren Umfeld als Moorobjekte. Jedes Moorobjekt wurde anhand von Luftbildern in einheitlich erscheinende Flächen unterteilt. Von jeder Fläche wurde eine vollständige Artenliste (Gefässpflanzen und Moose) erstellt. Die Flächen wurden durch Vergleich mit der Datenbank von R. Pantke (http://pages.unibas.ch/vegetation-ch/) Vegetationseinheiten zugeordnet. Die Einheiten wurden zudem in Hochmoorvegetation, Flachmoorvegetation (mit Feuchtwiesen) und Nichtmoorvegetation eingeteilt. Die Standortsbedingungen wurden ferner anhand der mittleren ökologischen Zeigerwerte nach Landolt für Nährstoffe und Feuchtigkeit charakterisiert.

In die Auswertung wurden die 53 Arten einbezogen, die in der Roten Liste der Blütenpflanzen und Farne der Schweiz (http://www.zdsf.ch/?page=roteliste) als invasive Neophyten bezeichnet wurden. Die verholzten Neophyten (Cornus sericea, Pinus strobus, Prunus serotina, Robinia pseudoacacia, Prunus laurocerasus), die in Mooren eher selten vorkommen, wurden für die Auswertung zu einer Gruppe zusammengefasst. Auch die nicht verholzte, aber hochwüchsige Staude Reynoutria japonica wurde derselben Gruppe zugeordnet. Zusätzlich wurde noch die neophytische Moosart Campylopus introflexus betrachtet, die seit wenigen Jahrzehnten stark in Ausbreitung begriffen ist. ${ }^{1}$

Die Verbreitungskarte in Abbildung 1 zeigt, dass vor allem die Moore tiefer Lagen von der Ausbreitung invasiver Neophyten betroffen sind. Nicht nur die Neophyten-Artenzahl pro Moorobjekt ist in tiefen Lagen höher (Abb. 1); die einzelnen Arten kommen auch innerhalb der Objekte häufiger vor, d.h. ein grösserer Teil der Moorfläche ist besiedelt (Daten nicht gezeigt).

Das Vorkommen der invasiven Neophyten hängt vom Moortyp ab. Invasive Neophyten wurden innerhalb der Objekte in 6\% der Hochmoorbereiche, 20\% der Flachmoorbereiche und $27 \%$ der Nichtmoorbereiche gefunden. Auch die mittlere Artenzahl und die lokale Häufigkeit der invasiven Neophyten waren in den Hochmoorflächen deutlich geringer als in Flachmooren und Nichtmooren.

Die Verteilung der Vorkommen auf die Vegetationseinheiten war je nach Art unterschiedlich (Tabelle. 1). Die meisten Arten kamen vor allem in Gehölzvegetation

\footnotetext{
${ }^{1}$ Urmi E, Schubiger-Bossard C, Schnyder N, Müller N, Küchler M, Hofmann H und Bisang I (2007) Zwei Jahrhunderte Bestandesentwicklung von Moosen in der Schweiz. Haupt, Bern.
} 
Abb. 1 Artenzahl invasiver Neophyten in den untersuchten Moorobjekten

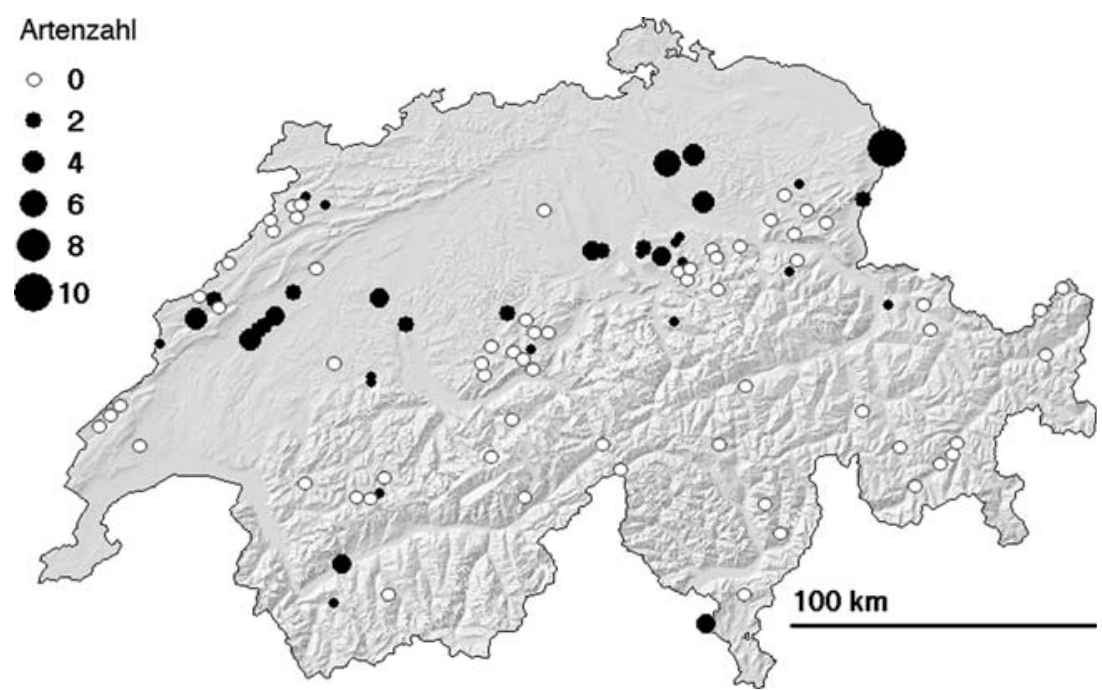

(W, G in Tabelle. 1) oder in den eutrophen Flachmoortypen (R, F) vor. Im Gegensatz hierzu hatte Campylopus introflexus seinen Schwerpunkt in offenen und bestockten Hochmooren $(\mathrm{H}, \mathrm{W}$ in Tabelle. 1). Diese Verbreitungsunterschiede hängen mit unterschiedlichen ökologischen Ansprüchen der Arten zusammen (Abb. 2): Die verholzten Neophyten kamen bei sehr verschiedenen Nährstoffverhältnissen vor, jedoch, wie auch Campylopus introflexus, nicht in den sehr nassen Flächen. Die krautigen Neophyten kamen nur in den nährstoffreicheren Flächen vor. Dabei konnten die Solidago-Arten und Impatiens parviflora auch nasse Flächen besiedeln, während das Vorkommen von Veronica filiformis und Heracleum mantegazzianum auf die trockeneren Flächen beschränkt war. Im Randbereich der Moorobjekte (N) wurden vereinzelt weitere invasive Neophytenarten wie Impatiens glandulifera, Epilobium ciliatum oder Parthenocissus quinquefolia gefunden; die
Wasserpflanze Elodea canadensis wurde im Bereich von Röhrichten (R) angetroffen.

Insgesamt zeigt die Untersuchung, dass invasive Neophyten vor allem in den relativ trockenen oder nährstoffreichen Bereichen der Moorobjekte zu finden sind. Um die Ausbreitung dieser Arten einzudämmen, sind vor allem Massnahmen zur Erhaltung oder Wiederherstellung des Wasser- und Nährstoffhaushalts notwendig.

Für den Moorschutz speziell relevant sind die Arten, welche von diesem Muster abweichen und auch in nassen oder nährstoffarmen Bereichen vorkommen.

Pinus strobus stellt als Art wahrscheinlich keine direkte Bedrohung für die Hochmoore dar. Sie wurde in Hochmooren angepflanzt, weil sie die dortigen Verhältnisse erträgt. Im Moment weist noch nichts darauf hin, dass sie die Hochmoorvegetation ohne Zutun des Menschen verdrängen würde.

Tabelle. 1 Stetigkeit der häufigsten invasiven Neophyten in verschiedenen Vegetationseinheiten: prozentualer Anteil besiedelter Kartierflächen pro Einheit

\begin{tabular}{lllllll}
\hline Vegetationseinheit & $\begin{array}{l}\text { Campylopus } \\
\text { introflexus }\end{array}$ & $\begin{array}{l}\text { Verholzte } \\
\text { Neophyten }\end{array}$ & $\begin{array}{l}\text { Solidago gigantea } \\
\text { \& S. canadensis }\end{array}$ & $\begin{array}{l}\text { Impatiens } \\
\text { parviflora }\end{array}$ & $\begin{array}{l}\text { Veronica } \\
\text { filiformis }\end{array}$ & $\begin{array}{c}\text { Heracleum } \\
\text { mantegazzianum }\end{array}$ \\
\hline H Bult- und Heidehochmoor & $1.3 \%$ & $0.3 \%$ & $0.0 \%$ & $0.0 \%$ & $0.0 \%$ & $0.0 \%$ \\
W Moorwald & $0.9 \%$ & $1.6 \%$ & $0.1 \%$ & $0.6 \%$ & $0.0 \%$ & $0.0 \%$ \\
U Übergangsmoor & $0.0 \%$ & $0.0 \%$ & $1.9 \%$ & $0.0 \%$ & $0.0 \%$ & $0.0 \%$ \\
A Saures Kleinseggenried & $0.3 \%$ & $0.1 \%$ & $0.0 \%$ & $0.0 \%$ & $0.0 \%$ & $0.0 \%$ \\
B Basenreiches Kleinseggenried & $0.0 \%$ & $0.0 \%$ & $0.9 \%$ & $0.1 \%$ & $0.0 \%$ & $0.0 \%$ \\
R Röhricht und Grossseggen & $0.3 \%$ & $0.3 \%$ & $5.6 \%$ & $0.6 \%$ & $0.5 \%$ & $0.0 \%$ \\
G Moorgebüsch & $0.3 \%$ & $2.0 \%$ & $5.3 \%$ & $4.0 \%$ & $0.3 \%$ & $0.0 \%$ \\
F Spierstaudenflur & $0.0 \%$ & $0.0 \%$ & $5.2 \%$ & $0.3 \%$ & $0.8 \%$ & $1.0 \%$ \\
C Nasswiesen & $0.03 \%$ & $0.0 \%$ & $1.0 \%$ & $0.6 \%$ & $0.2 \%$ & $0.1 \%$ \\
N Nichtmoor & $0.1 \%$ & $0.6 \%$ & $1.1 \%$ & $0.7 \%$ & $0.2 \%$ \\
\hline
\end{tabular}



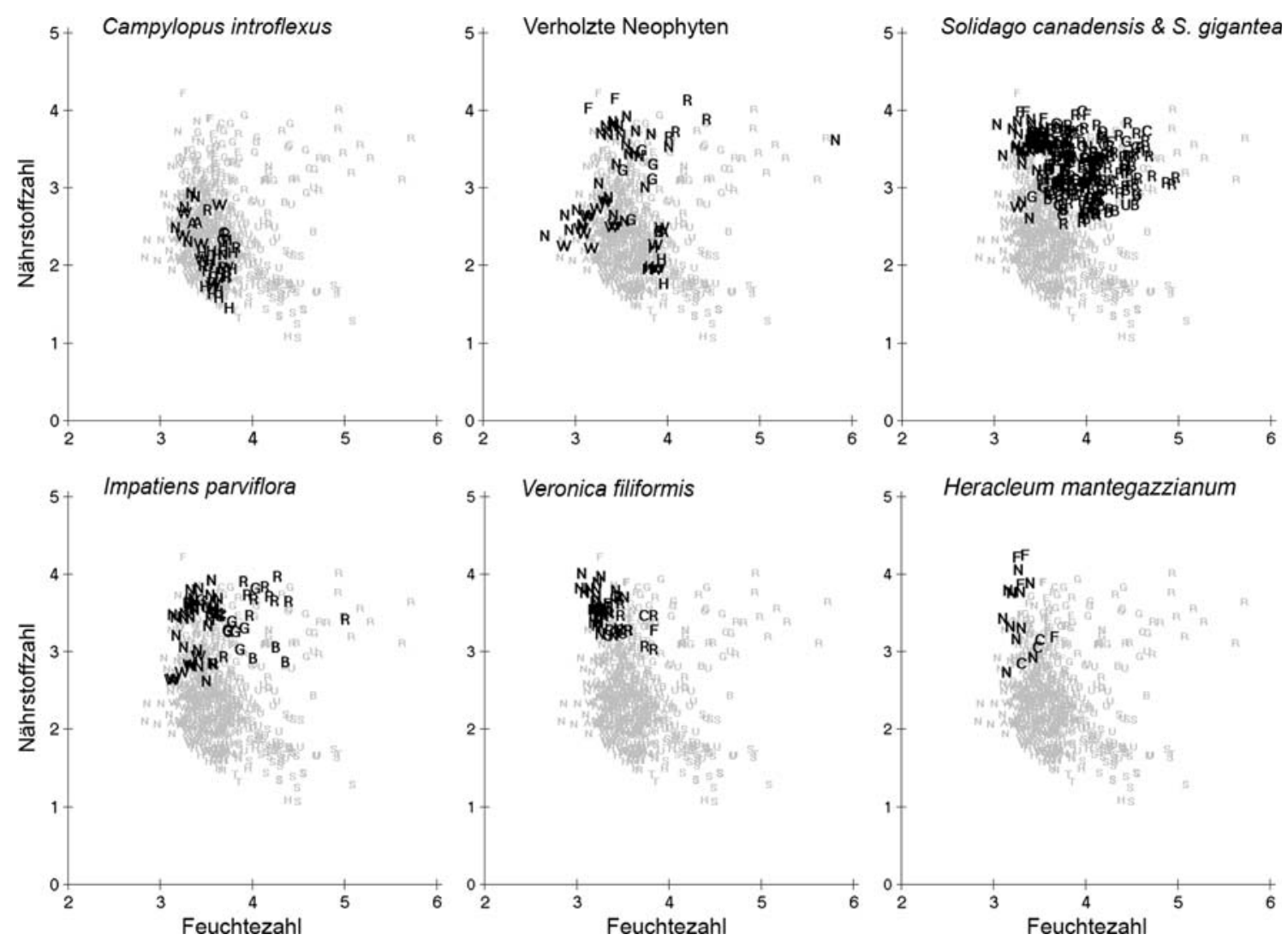

Abb. 2 Vorkommen von Neophyten-Arten in Abhängigkeit der Feuchte- und der Nährstoffzahl. Zeigerwerte nach Landolt, angepasst für Moorbiotope (Skala von 1 bis 6). Die schwarzen Symbole bezeichnen Aufnahmen mit der betreffenden Art und deren Zugehörigkeit zu einer
Vegetationseinheit (siehe Tabelle. 1). Die kleineren, grauen Symbole stellen eine zufällige Auswahl von Aufnahmen aus dem Datensatz (Aufnahmen der Wirkungskontrolle Moorschutz) dar

trifft für Solidago gigantea, Solidago canadensis, Impatiens parviflora und das Moos Campylopus introflexus zu. Auch Reynoutria japonica zeigt in gewissen Mooren eigendynamisches Verhalten (z.B. am Bodenseeufer oder in der Magadino-Ebene). Diese Arten müssen als mögliche Bedrohung der Moorvegetation betrachtet werden, welche auch bei einem verbesserten Wasserhaushalt bestehen bleibt. Hier dürften neben der rigorosen Vermeidung weiterer Ansiedlungen gezielte Massnahmen unverzichtbar sein.

Bei einigen Arten weisen die Resultate dieser Studie auf eine Eigendynamik der Ausbreitung in den Mooren hin. Das 